\title{
Quantum Synchronization Blockade: Energy Quantization hinders Synchronization of Identical Oscillators
}

\author{
Niels Lörch, ${ }^{1}$ Simon E. Nigg, ${ }^{1}$ Andreas Nunnenkamp, ${ }^{2}$ Rakesh P. Tiwari,,${ }^{1,3}$ and Christoph Bruder ${ }^{1}$ \\ ${ }^{1}$ Department of Physics, University of Basel, Klingelbergstrasse 82, CH-4056 Basel, Switzerland \\ ${ }^{2}$ Cavendish Laboratory, University of Cambridge, Cambridge CB3 OHE, United Kingdom \\ ${ }^{3}$ Department of Physics, McGill University, Montreal, Quebec, Canada
}

(Dated: June 23, 2017)

\begin{abstract}
Classically, the tendency towards spontaneous synchronization is strongest if the natural frequencies of the self-oscillators are as close as possible. We show that this wisdom fails in the deep quantum regime, where the uncertainty of amplitude narrows down to the level of single quanta. Under these circumstances identical self-oscillators cannot synchronize and detuning their frequencies can actually help synchronization. The effect can be understood in a simple picture: Interaction requires an exchange of energy. In the quantum regime, the possible quanta of energy are discrete. If the extractable energy of one oscillator does not exactly match the amount the second oscillator may absorb, interaction, and thereby synchronization is blocked. We demonstrate this effect, which we coin quantum synchronization blockade, in the minimal example of two Kerr-type self-oscillators and predict consequences for small oscillator networks, where synchronization between blocked oscillators can be mediated via a detuned oscillator. We also propose concrete implementations with superconducting circuits and trapped ions. This paves the way for investigations of new quantum synchronization phenomena in oscillator networks both theoretically and experimentally.
\end{abstract}

Coupled self-oscillating systems can spontaneously synchronize, i.e., align their phase and frequency. This phenomenon $[1,2]$ is observed in a multitude of systems, ranging from the spontaneous blinking of fireflies in unison to the firing of neurons in the human brain, and technical applications such as lasers.

The laser is a well-known example of a quantum system that is described as a self-osillator. However, its steady state far above threshold settles into a coherent state, which is essentially classical $[3,4]$. Therefore, its synchronization behavior could so far be fully described within a semiclassical picture [5, 6], which allows for efficient simulations. Along this line, powerful methods have been developed capable of describing large quantum oscillator arrays, such as complex lasing media [7, 8], arrays of optomechanical systems [9, 10] and polariton condensates [11-14].

The rapid experimental progress [15-18] in the control of quantum oscillators and in the engineering of their dissipative reservoirs [19-25] is opening the opportunity to study synchronization deep in the quantum regime, where only a few energy states are populated [26-37]. In this regime semiclassical methods can fail [27] and anharmonicity on the level of single quanta has been identified [34] as a crucial ingredient to demonstrate quantum effects in synchronization.

In this paper, we discuss a new class of effects in the synchronization of quantum self-oscillators: For the simplest case of two coupled self-oscillators we find that a finite frequency detuning between different oscillators may enable synchronization in the quantum regime, while synchronization between (nearly) identical self-oscillators is suppressed. Relatedly, two identical oscillators of different amplitude are found to synchronize better than oscillators of the same amplitude. These findings are in stark contrast to our classical expectation and elude any semiclassical model. The effect generalizes to oscillator networks: identical oscillators, while unable (a)
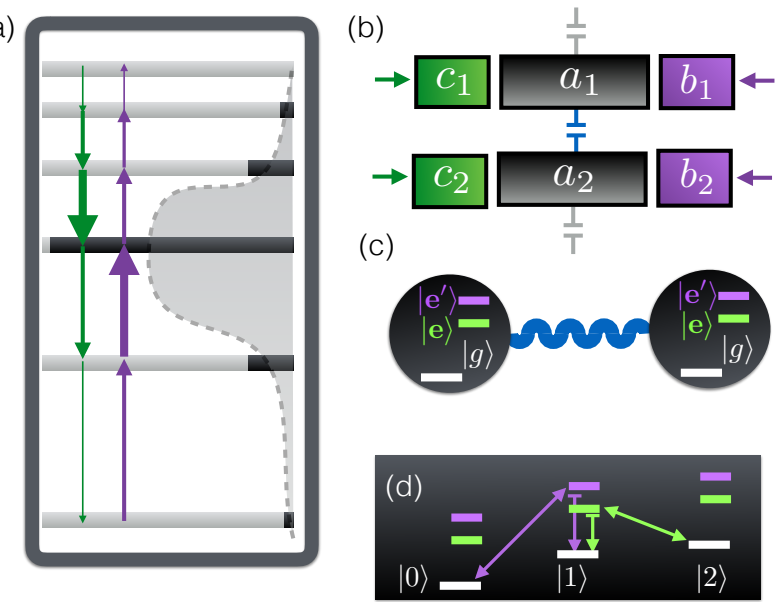

Figure 1. (a) Illustration of an anharmonic oscillator level structure (grey) with nonlinear amplification (purple) and damping (green) tuned such that a particular Fock state (here the state $|2\rangle$ ) is stabilized. The relative thickness of the arrows indicates the transition rate; steady-state population probabilities are depicted in black. The corresponding classical system would have a continuous energy distribution, which is sketched in grey. (b) Implementation with an array of superconducting anharmonic oscillators driven by an amplification cavity (purple) and a damping cavity (green), see main text. (c) Implementation with trapped ions with excited states transition between ground state $|g\rangle$ and excited states $|e\rangle,\left|e^{\prime}\right\rangle$ enabling respectively sideband cooling and sideband amplification of motion as depicted in (d).

to synchronize directly, can synchronize via a third detuned oscillator. We propose possible implementations in a network of superconducting circuits $[16,17,38]$ or using trapped ions $[15,39]$ to demonstrate the effect experimentally. Our study opens up a novel regime of synchronization with genuine quantum features that can be observed with state-of-the- 
art quantum hardware.

Quantum Model of the System.- We consider a network of anharmonic oscillators each described by the Hamiltonian

$$
H=\omega a^{\dagger} a-K\left(a^{\dagger}\right)^{2} a^{2}
$$

where $a$ is a bosonic annihilation operator, $\omega$ is the natural frequency of the oscillator, and the Kerr parameter $K$ quantifies the anharmonicity. Crucially, the quantum oscillators are subject to dissipation which drives them into self-sustained oscillations (limit cycles). In the framework of open quantum systems this is modeled with a Lindblad operator $\mathcal{L}=\mathcal{L}^{(-)}+\mathcal{L}^{(+)}$ consisting of damping $\mathcal{L}^{(-)}$and amplification $\mathcal{L}^{(+)}$.

To unravel quantum signatures most clearly, we aim for a narrow distribution of Fock states in steady state, ideally a single Fock state. One way to achieve [40] this is with highly nonlinear dissipators

$$
\begin{aligned}
& \mathcal{L}^{+}=\frac{\gamma_{+}}{2} \sum_{n} f_{+}(n) \mathcal{D}[\sqrt{n}|n\rangle\langle n-1|], \\
& \mathcal{L}^{-}=\frac{\gamma_{-}}{2} \sum_{n} f_{-}(n) \mathcal{D}[\sqrt{n}|n-1\rangle\langle n|],
\end{aligned}
$$

where the individual terms induce transitions from Fock state $|n\rangle$ to Fock state $|n-1\rangle$. The transition rates $\propto f_{+}\left(f_{-}\right)$are highly peaked just below (above) the desired Fock state [41], as illustrated in Fig. 1 (a). Our physical implementation described below results in

$$
f_{ \pm}(n)=\frac{\sigma_{ \pm}^{2}}{\left(n-n_{ \pm}\right)^{2}+\sigma_{ \pm}^{2}},
$$

where $n_{ \pm}$and $\sigma_{ \pm}$are mean and variance of the Lorentzian. Choosing $n_{+}$near an integer $n_{0}$ and $n_{-}$near $n_{0}+1$ stabilizes that particular Fock state $\left|n_{0}\right\rangle$, where a high fidelity is achieved if both $\sigma_{-}, \sigma_{+} \ll 1$. For simplicity, we choose from here on $\sigma_{ \pm}=\sigma, \gamma_{ \pm}=\gamma$, and $n_{-}=n_{+}+1$.

This corresponds to the extreme quantum limit of selfoscillations, where the energy distribution is so sharp that only a single Fock state is populated. Therefore, due to the phasenumber uncertainty, the phase must be in a superposition of all phases. In comparison, the state of an ordinary laser, as described by an incoherent mixture of coherent states, also has an undefined phase of classical uncertainty but not as a result of superposition.

The quantum master equation for the density matrix $\rho$ of a complete network of such self-oscillators (numbered with index $j$ ) that are reactively coupled, is given by

$$
\dot{\rho}=-i\left[\sum_{j} H_{j}+V, \rho\right]+\sum_{j} \mathcal{L}_{j} \rho, \quad V=\sum_{j, k} C_{j k} a_{j}^{\dagger} a_{k},
$$

where the commutator between $A$ and $B$ is denoted as $[A, B]$ and the coupling matrix associated with the interaction $V$ fulfills $C_{j k}=C_{j k}^{*}$ and $C_{j j}=0$.

Classical Model of the System.- We will now introduce the corresponding classical description to be able to compare the
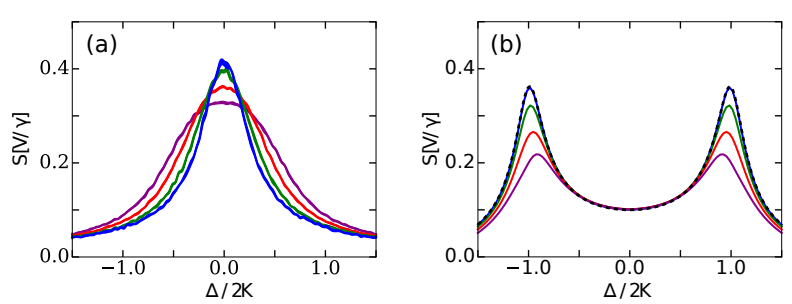

Figure 2. Synchronization measure $S$ calculated using Eq. (6) as a function of the detuning $\Delta$ between two oscillators. All other parameters are identical for both oscillators. Panel (a) shows the result of classical Monte-Carlo simulations of Eq. (5), where the width of the line indicates the statistical error. Panel (b) are results from the numerical steady-state solution of the quantum master equation (4). Classical parameters: $\gamma_{T} n_{t}=0.1 \gamma, \sigma=0.2, n_{+}=$ $2, n_{-}=3, K=2 \gamma, V=0.1 \gamma \cdot\left(\frac{1}{6}, \frac{1}{4}, \frac{3}{8}, \frac{1}{2}\right)$. Quantum parameters: $\sigma=0.2, n_{+}=2, n_{-}=3, K=10 \gamma, V=\gamma \cdot(0.05,0.2,0.35,0.5)$. In both panels $\gamma_{1 \pm}=\gamma_{2 \pm}=\gamma$. In first-order perturbation theory (dashed black line), the height of the maximal peak is proportional to the coupling $V$ [46]. Plotting $S$ in units of $V$ for the numerical results, the height of the peak decreases with increasing $V$, where higher-order effects play a role. The noise level is chosen such that the effect of the thermal noise in panel (a) is approximately as strong as the quantum noise in panel (b).

quantum system to its classical limit. The self-oscillators in Eqs. (4) can be described by the Langevin equations

$$
\dot{\alpha}_{j}=-\left(i \Omega_{j}\left(\alpha_{j}\right) \alpha_{j}+\frac{\Gamma_{j}\left(\alpha_{j}\right)}{2}\right) \alpha_{j}-i C_{j k} \alpha_{k}+\eta_{j}
$$

with the classical oscillator amplitudes $\alpha_{j}$. Here $\Omega_{j}(\alpha)=$ $\omega_{j}-2 K_{j}|\alpha|^{2}$ and $\Gamma_{j}(\alpha)=\gamma_{j-} f_{j-}\left(|\alpha|^{2}\right)-\gamma_{j-} f_{j+}\left(|\alpha|^{2}\right)$ are the amplitude-dependent frequency and damping rate of the $j$-th oscillator, and $C_{j k}$ is the coupling matrix from Eq. (4). Finally $\eta_{j}$ is a white-noise process with correlator $\left\langle\eta_{k}(t) \eta_{j}\left(t^{\prime}\right)\right\rangle=$ $\delta_{k j} \delta\left(t-t^{\prime}\right) \gamma_{T} n_{T}$ where $n_{T}$ is the thermal bath occupation and $\gamma_{T}$ is the coupling rate to the bath. For conceptual clarity we adopt here the fully classical picture neglecting quantum noise induced by the damping terms $\propto \gamma_{+}, \gamma_{-}$. Our main conclusions are not affected by this choice. A derivation of the semiclassical equations including quantum noise can be found in Ref. [42].

Synchronization Measures.- To quantify synchronization between two oscillators we consider the distribution $P(\phi)=$ $\iint_{0}^{2 \pi} \mathrm{d} \phi_{1} \mathrm{~d} \phi_{2} \delta\left(\phi_{1}-\phi_{2}-\phi\right) p\left(\phi_{1}, \phi_{2}\right)$ of their relative phase $\phi$. For the quantum steady state $\rho_{s s}$, we define $p\left(\phi_{1}, \phi_{2}\right)=$ $\left\langle\phi_{1}, \phi_{2}\left|\rho_{s s}\right| \phi_{1}, \phi_{2}\right\rangle$ with phase states $|\phi\rangle=\frac{1}{2 \pi} \sum_{n=0}^{\infty} e^{i n \phi}|n\rangle$ [43]. For the classical case, we define $p\left(\phi_{1}, \phi_{2}\right)$ as the probability of $\left(\alpha_{1}, \alpha_{2}\right)$ to have phases $\left(\phi_{1}, \phi_{2}\right)$ in the steady state of Eq. (5). In both cases we choose the synchronization measure [44, 45]

$$
S=2 \pi \max _{\phi}[P(\phi)]-1,
$$

i.e. a scaled maximum of the relative phase distribution.

Quantum Synchronization Blockade.- We now consider the self-oscillator depicted in Fig. 1 (a) coupled to another such 
self-oscillator with all identical parameters, except for the natural frequencies which are detuned by $\Delta=\omega_{1}-\omega_{2}$. According to classical intuition, the strongest tendency to synchronize as a function of $\Delta$ as measured by (6) is always achieved at $\Delta=0$, where both oscillators are identical. This picture is confirmed by the numerical solution of Eq. (5), which is presented in Fig. 2 (a). It is consistent with analytical results obtained in a study of exciton-polariton condensates [12], corresponding to the zero-temperature limit of Eq. (5).

The classical intuition is not valid in the quantum system described by Eqs. (4). We investigate the same setup with parameters deep in the quantum regime, where the limit cycle is essentially stabilized to a single Fock state $\left|n_{0}\right\rangle$. The numerical result depicted in Fig. 2 (b) converge with decreasing coupling strengths to an analytical perturbation theory derived in [46]. The phase synchronization measure is suppressed at $\Delta=0$, where $S$ has a local minimum. Instead, phase synchronization is now maximal at two peaks at $\Delta= \pm 2 \mathrm{~K}$.

We call this phenomenon the quantum synchronization blockade, as it only occurs deep in the quantum regime, where almost all population is stabilized to a single Fock state. The transition from quantum to classical is visualized in Fig. 3 (a): For a narrow Fock distribution around $\sigma=0.2$ the two maxima of synchronization appear at $\Delta= \pm 2 K$, as just discussed. With increasing width $\sigma$, the maxima merge to one broad resonance around $\Delta=0$, as classically expected.

In a second scenario we consider self-oscillators of identical frequency, now differing only in the amplitude $\bar{n}$ at which they are stabilized. Oscillator 1 is stabilized to an integer $\bar{n}=n_{0}$ as before, while the amplitude $\bar{n}$ of oscillator 2 is varied continuously. The result is shown in Fig. 3 (b): In the quantum regime of small $\sigma$ synchronization is maximal at $\bar{n}_{2}=\bar{n}_{1} \pm 1$, i.e. oscillators with a finite difference in amplitude amplitude are most likely to synchronize. Again the classical intuition, that maximal synchronization will be present for identical oscillators with $\bar{n}_{1}=\bar{n}_{2}$, is confirmed in the classical regime of larger $\sigma$.

Thus, in contrast to classical expectation, synchronization of two quantum oscillators can be enhanced by making the oscillators more heterogeneous via detuning their frequency or via a mismatch in their amplitude. The result can be explained as follows: For two oscillators to interact efficiently, the process $\propto a_{k}^{\dagger} a_{j}$ of exchanging one excitation must be resonant by conserving energy. For oscillator $j$ in state $\left|n_{j}\right\rangle$ to transfer an excitation to oscillator $k$ initially in state $\left|n_{k}\right\rangle$ it is required that $E\left(\left|n_{j}, n_{k}\right\rangle\right)=E\left(\left|n_{j}-1, n_{k}+1\right\rangle\right)$. Writing the energy as $E\left(\left|n_{j}, n_{k}\right\rangle\right)=\left\langle n_{j}, n_{k}|H| n_{j}, n_{k}\right\rangle=\omega_{j} n_{j}+\omega_{k} n_{k}-K\left(n_{j}^{2}+\right.$ $n_{k}^{2}-n_{j}-n_{k}$ ), this leads to the two resonances

$$
\Delta+2 K \Delta n \pm 2 K=0,
$$

where $\Delta=\omega_{j}-\omega_{k}$ and $\Delta n=n_{j}-n_{k}$. This resonance condition is one of the main results of our paper and is illustrated in Fig. 3 (c), showing the synchronization measure as a function of both $\Delta$ and $\Delta n$ in the quantum regime. For an illustration of the resonance condition in the case of identical oscillators, see [46].
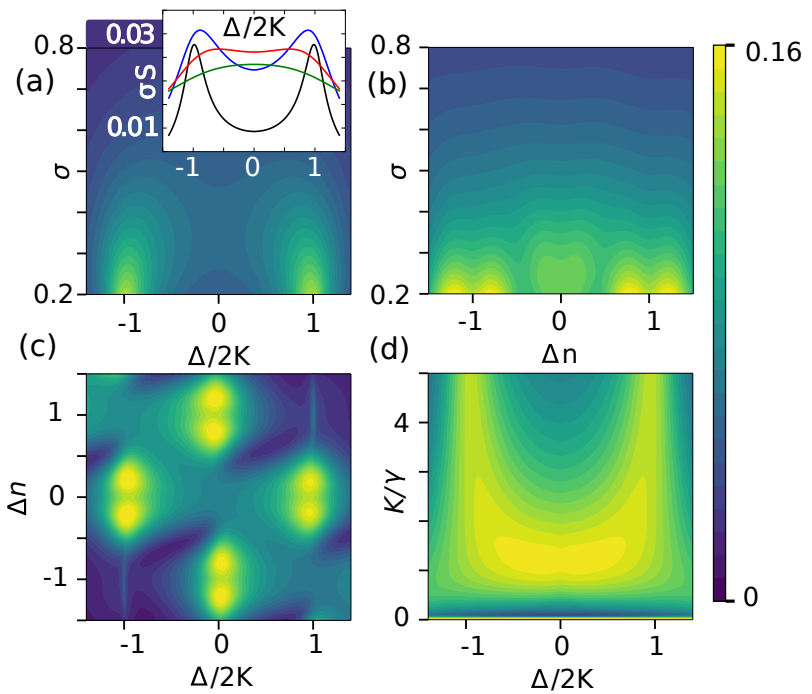

Figure 3. Plots of synchronization measure $S$ from Eq. (2). (a) Identical oscillators differing only in frequency. Inset shows cuts of $S$ scaled by $\sigma$ at $\sigma=0.2,0.4,0.6,0.8$ in black, blue, red, green. (b) Identical oscillators differing only in amplitude. (c) Overview of these resonances in $\Delta n$ and $\Delta$. (d) Resonances as a function of the Kerr nonlinearity $K$. Parameters: In the upper panels $n_{1}^{+} \equiv 4$, $n_{j}^{-}=n_{j}^{+}+1, V=0.1 \gamma, K=\gamma / \sigma_{ \pm}, \gamma_{1_{ \pm}}=\gamma_{2 \pm}=\gamma$. In (a) $n_{2}^{+}=4$ and in (b) $\omega_{1}=\omega_{2}$. In the lower panels $\sigma_{ \pm}=0.2$ and all other parameters are as above.

Equation (7) includes an offset of $2 K$ stemming from the mismatch of energy in the exchange of a single quantum of energy described above. Classically, arbitrarily small quanta may be exchanged, so that the offset does not exist. For the oscillators to interact efficiently (and thereby to synchronize), an upward transition of oscillator $k$ must be resonant with a downward transition of oscillator $j$, or vice versa.

Fig. 3 (d) shows how the resonances may be resolved for increasing $K$. For $K=0$ we have the situation of resonant harmonic oscillators [27], where $P(\phi)$ is a bimodal distribution at $K=0$. Increasing $K$ first leads to a suppression of the resonance and then to a splitting at $\Delta= \pm 2 K$. In this regime only one maximum of $P(\phi)$ survives.

Oscillator Networks.- Having established the quantum synchronization blockade, we now use this understanding to explore consequences for networks of oscillators. In the following, we focus on small networks, as these are easiest to implement experimentally. Consider the three-oscillator network depicted in the inset of Fig. 4 (c), where two identical oscillators $A$ and $B$ are coupled indirectly by connecting them with coupling strength $V_{1}$ to an oscillator $C$ which has a relative detuning $\Delta$ with respect to $A$ and $B$. We first look at the case where the direct coupling $V_{2}$ between the two identical oscillators is zero. As shown in Fig. 4 (a), resonances occur at $\Delta= \pm 2 K$ for the synchronization between detuned oscillators, as expected from the two-oscillator case. Figure 4 (b) shows that identical oscillators can now synchronize via mediation of the detuned oscillator, again with resonances at $\Delta= \pm 2 \mathrm{~K}$. In this way, the synchronization blockade can be lifted. This 

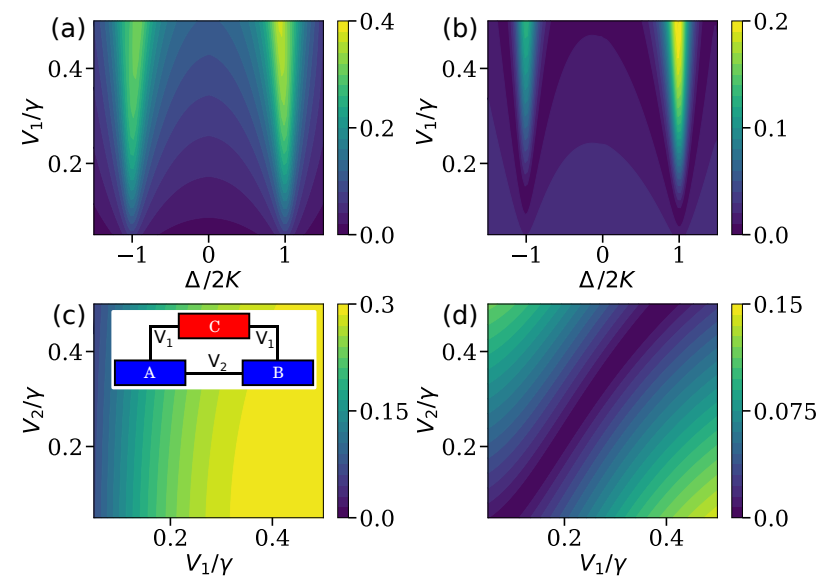

Figure 4. Synchronization measure $S$ for the network of three oscillators depicted in the inset of panel (c). In the upper panels two identical oscillators are connected indirectly via an oscillator $C$ with detuning $\Delta$, while the direct link $V_{2}=0$. In the lower panel the direct link $V_{2}$ is also turned on and $\Delta \equiv 2 K$. In both all panels $n_{1}^{+} \equiv 1, n_{j}^{-}=n_{j}^{+}+1, K=10 \gamma, \gamma_{1 \pm}=\gamma_{2 \pm}=\gamma$. In both rows the left panel shows synchronization between different oscillators and the right panel between identical oscillators. We conclude from the upper panels that identical oscillators can be synchronized indirectly via a detuned oscillator. The lower panels indicate that increasing the direct coupling can even decrease synchronization for a strong enough indirect link: In (c) the contour lines bend to the right, while in (d) a strip of suppressed synchronization appears.

finding is also confirmed for the larger network of four oscillators, where two pairs of identical oscillators are connected in a ring in alternating order, see [46].

Conversely, as shown in Figs. 4 (c) and (d), turning on a coupling $V_{2}$ between the identical oscillators can suppress synchronization. This effect is most pronounced for identical oscillators, for which a strip of suppressed synchronization appears along $V_{1} \propto V_{2}$.

Implementation.- Nonlinear damping of the form (3) can be naturally achieved for an anharmonic oscillator mode $a$ coupled to a linear cavity mode $c$ by coupling the number $\propto c^{\dagger} c$ of the cavity to the quadrature $a+a^{\dagger}$. Driving the cavity on the red (blue) sideband will lead to a positive (negative) damping [40, 42]. Due to the anharmonic level structure the position of the sidebands depends on the oscillator amplitude. Therefore, in contrast to ordinary sideband cooling, the strength of both damping and amplification depends nonlinearly on the oscillation amplitude.

In a rotating frame of the cavity drive $E$ the Hamiltonian is given by $H_{c}=-\delta c^{\dagger} c+E\left(c+c^{\dagger}\right)+g_{0} c^{\dagger} c\left(a+a^{\dagger}\right)$, where $\delta$ is the laser detuning and $g_{0}$ is the coupling rate. Defining $g=g_{0} \sqrt{\left\langle c^{\dagger} c\right\rangle}$ this can be linearized in the regime of large amplitudes $\left(\left\langle c^{\dagger} c\right\rangle \gg 1\right)$ as $H_{c}=-\delta c^{\dagger} c+g\left(a^{\dagger}+a\right)\left(c+c^{\dagger}\right)$. Assuming that the cavity decay rate $\kappa$ fulfills $g \ll \kappa$ such that the cavity can be adiabatically eliminated, the parameters of our dissipators (2) are approximately given by [40] $\gamma=4 g^{2} / \kappa$, $\sigma=\kappa / 8 K$, and $n_{ \pm}= \pm\left(\delta_{ \pm}-\omega_{0}\right) / 2 K$.

Thus, to achieve small $\sigma$ and thereby stabilize a Fock state, a large anharmonicity to cavity noise ratio $K / \kappa \gtrsim 1$ is required. As depicted in Fig. 3 (d) and reflected in the perturbation theory from [46], also $K / \gamma \gtrsim 1$ is necessary. As $\gamma=4 g^{2} / \kappa$ and $g \ll \kappa$, we have the hierarchy $\kappa>\gamma$ and therefore only $K / K \gtrsim 1$ remains as the feasibility condition for our specific implementation. This condition is a challenging requirement on the experimental setup. For instance, optomechanical systems, while highly coherent, still lack strong enough anharmonicity. While this may be overcome in the future e.g. using auxiliary coupling to a Cooper pair transistor [47], we propose an implementation using superconducting circuits and, alternatively, trapped ions. In both platforms a large anharmonicity $K \gg \kappa, \gamma$ can be achieved with state-of-the-art technology.

An implementation using superconducting circuits is schematically depicted in Fig. 1 (b) for the case of two capacitively coupled self-oscillators $a_{j}$. To implement larger networks, the array can be extended along the greyed out coupling capacitors. One choice of self-oscillators are transmon qubits [16] which are sufficiently anharmonic, while offering a long enough coherence time. The auxiliary cavities for amplification $\left(b_{j}\right)$ and damping $\left(c_{j}\right)$, are coupled to the self-oscillator via an interaction of optomechanical form, $c^{\dagger} c\left(a+a^{\dagger}\right)$. This can be brought about by embedding a SQUID in the auxiliary cavity [38]. The particular Lorentzian form (3) was assumed as a concrete example, but the scheme is quite general, i.e. any other setup with both nonlinear damping and amplification could be used; any other means of Fock state stabilization such as $[23,48,49]$ will be equally suitable for our purposes.

An implementation using trapped ions is depicted in Fig. 1 (c). Ions trapped in adjacent highly anharmonic potentials [39] can become self-oscillators with dissipation engineered as follows: The roles of the cavities for amplification and damping are now played by the internal level structure of the ion, with one transition driven on the blue sideband and another transition on the red sideband. The use of two transitions is similar to the schemes $[27,44]$ to implement self-oscillators with ions. The ions are naturally coupled via the Coulomb interaction $[44,50,51]$.

We note that to observe the effect presented here each node of the network needs to have an anharmonic spectrum consisting of at least three levels, excluding arrays of harmonic oscillators or qubits [46].

Conclusion and Outlook.- To conclude, we have described a novel effect referred to as the quantum synchronization blockade, which prevents identical nonlinear oscillators from synchronizing deep in the quantum regime. This is in stark contrast to the classical regime, where oscillators synchronize best when on resonance. Complementarily we have demonstrated that detuned auxiliary oscillators can lift this blockade by indirectly mediating synchronization between identical oscillators. These effects will be observable in state-of-the-art quantum systems such as superconducting circuits and trapped ions, for which we have proposed concrete implementations. Our article thus opens a new perspective for the exploration of synchronization in Bose-Hubbard-van der 
Pol-type networks.

Acknowledgments.- We would like to acknowledge helpful discussions with A. H. Safavi-Naeini and S. M. Girvin. This work was financially supported by the Swiss SNF and the NCCR Quantum Science and Technology. A.N. holds a University Research Fellowship from the Royal Society and acknowledges support from the Winton Programme for the Physics of Sustainability. All quantum steady-state solutions were obtained in QuTiP [52] and the classical Monte-Carlo trajectories were performed in Julia [53]. This work was supported by the European Union's Horizon 2020 research and innovation programme under grant agreement No 732894 (FET Proactive HOT).

[1] A. Pikovsky, M. Rosenblum, and J. Kurths, Synchronization: a universal concept in nonlinear sciences, Vol. 12 (Cambridge university press, 2003).

[2] A. Balanov, N. Janson, D. Postnov, and O. Sosnovtseva, Synchronization: From Simple to Complex (Springer, 2009).

[3] C. Gardiner and P. Zoller, Quantum Noise (Springer, 2004).

[4] D. F. Walls and G. J. Milburn, Quantum Optics (Springer, 2008).

[5] W. Schleich and M. O. Scully, Phys. Rev. A 37, 1261 (1988).

[6] W. Schleich, M. O. Scully, and H. G. von Garssen, Phys. Rev. A 37, 3010 (1988).

[7] H. E. Türeci, A. D. Stone, and B. Collier, Phys. Rev. A 74, 043822 (2006).

[8] O. Malik, K. G. Makris, and H. E. Türeci, Phys. Rev. A 92, 063829 (2015).

[9] M. Ludwig and F. Marquardt, Phys. Rev. Lett. 111, 073603 (2013).

[10] T. Weiss, A. Kronwald, and F. Marquardt, New J. Phys. 18, 013043 (2016).

[11] P. R. Eastham, Phys. Rev. B 78, 035319 (2008).

[12] M. Wouters, Phys. Rev. B 77, 121302 (2008).

[13] S. Khan and H. E. Türeci, Phys. Rev. A 94, 053856 (2016).

[14] L. M. Sieberer, M. Buchhold, and S. Diehl, Reports on Progress in Physics 79, 096001 (2016).

[15] D. Leibfried, R. Blatt, C. Monroe, and D. Wineland, Rev. Mod. Phys. 75, 281 (2003).

[16] J. Koch, T. M. Yu, J. Gambetta, A. A. Houck, D. I. Schuster, J. Majer, A. Blais, M. H. Devoret, S. M. Girvin, and R. J. Schoelkopf, Phys. Rev. A 76, 042319 (2007).

[17] R. J. Schoelkopf and S. M. Girvin, Nature 451, 664 (2008).

[18] M. Dykman, Fluctuating Nonlinear Oscillators: From Nanomechanics to Quantum Superconducting Circuits (Oxford University Press, 2012).

[19] J. F. Poyatos, J. I. Cirac, and P. Zoller, Phys. Rev. Lett. 77, 4728 (1996).

[20] C. J. Myatt, B. E. King, Q. A. Turchette, C. A. Sackett, D. Kielpinski, W. M. Itano, C. Monroe, and D. J. Wineland, Nature 403, 269 (2000).

[21] K. Vahala, M. Herrmann, S. Knünz, V. Batteiger, G. Saathoff, T. Hänsch, and T. Udem, Nature Physics 5, 682 (2009).

[22] D. Kienzler, H.-Y. Lo, B. Keitch, L. de Clercq, F. Leupold, F. Lindenfelser, M. Marinelli, V. Negnevitsky, and J. Home, Science 347, 53 (2015).

[23] E. T. Holland, B. Vlastakis, R. W. Heeres, M. J. Reagor, U. Vool, Z. Leghtas, L. Frunzio, G. Kirchmair, M. H. Devoret,
M. Mirrahimi, and R. J. Schoelkopf, Phys. Rev. Lett. 115, 180501 (2015).

[24] J. Roßnagel, S. T. Dawkins, K. N. Tolazzi, O. Abah, E. Lutz, F. Schmidt-Kaler, and K. Singer, Science 352, 325 (2016).

[25] M. Fitzpatrick, N. M. Sundaresan, A. C. Y. Li, J. Koch, and A. A. Houck, Phys. Rev. X 7, 011016 (2017).

[26] A. Mari, A. Farace, N. Didier, V. Giovannetti, and R. Fazio, Phys. Rev. Lett. 111, 103605 (2013).

[27] T. E. Lee and H. R. Sadeghpour, Phys. Rev. Lett. 111, 234101 (2013).

[28] T. E. Lee, C.-K. Chan, and S. Wang, Phys. Rev. E 89, 022913 (2014).

[29] S. Walter, A. Nunnenkamp, and C. Bruder, Phys. Rev. Lett. 112, 094102 (2014).

[30] S. Walter, A. Nunnenkamp, and C. Bruder, Ann. Phys. 527, 131 (2014).

[31] M. Xu, D. A. Tieri, E. C. Fine, J. K. Thompson, and M. J. Holland, Phys. Rev. Lett. 113, 154101 (2014).

[32] M. Xu and M. J. Holland, Phys. Rev. Lett. 114, 103601 (2015).

[33] V. Ameri, M. Eghbali-Arani, A. Mari, A. Farace, F. Kheirandish, V. Giovannetti, and R. Fazio, Phys. Rev. A 91, 012301 (2015).

[34] N. Lörch, E. Amitai, A. Nunnenkamp, and C. Bruder, Phys. Rev. Lett. 117, 073601 (2016).

[35] T. Weiss, S. Walter, and F. Marquardt, Phys. Rev. A 95, 041802 (2017).

[36] F. Galve, G. L. Giorgi, and R. Zambrini, arXiv:1610.05060 (2016).

[37] G. L. Giorgi, F. Galve, and R. Zambrini, Phys. Rev. A 94, 052121 (2016).

[38] J. R. Johansson, G. Johansson, and F. Nori, Phys. Rev. A 90, 053833 (2014).

[39] L. E. de Clercq, H.-Y. Lo, M. Marinelli, D. Nadlinger, R. Oswald, V. Negnevitsky, D. Kienzler, B. Keitch, and J. P. Home, Phys. Rev. Lett. 116, 080502 (2016).

[40] S. Rips, M. Kiffner, I. Wilson-Rae, and M. J. Hartmann, New J. Phys. 14, 023042 (2012).

[41] Note that the dissipators in Eq. (2) have no off-diagonal elements between each other in the Lindblad equation [40]. Depending on the concrete system and parameters these coherences may be present. The effects we want to show exist in both cases though, so we use this simpler model here.

[42] M. Grimm, C. Bruder, and N. Lörch, J. Opt. 18, 094004 (2016).

[43] R. Barak and Y. Ben-Aryeh, J. Opt. B Quantum Semiclassical Opt. 7, 123 (2005).

[44] M. R. Hush, W. Li, S. Genway, I. Lesanovsky, and A. D. Armour, Phys. Rev. A 91, 061401 (2015).

[45] C. Davis-Tilley and A. D. Armour, Phys. Rev. A 94, 063819 (2016).

[46] See Supplemental Material.

[47] A. J. Rimberg, M. P. Blencowe, A. D. Armour, and P. D. Nation, New J. Phys. 16, 055008 (2014).

[48] J. R. Souquet and A. A. Clerk, Phys. Rev. A 93, 060301(R) (2016).

[49] R. Ma, C. Owens, A. Houck, D. I. Schuster, and J. Simon, Phys. Rev. A 95, 043811 (2017).

[50] K. Brown, C. Ospelkaus, Y. Colombe, A. Wilson, D. Leibfried, and D. Wineland, Nature 471, 196 (2011).

[51] M. Harlander, R. Lechner, M. Brownnutt, R. Blatt, and W. Hänsel, Nature 471, 200 (2011).

[52] J. Johansson, P. Nation, and F. Nori, Comput. Phys. Commun. 184, 1234 (2013).

[53] J. Bezanson, A. Edelman, S. Karpinski, and V. B. Shah, SIAM Review 59, 65 (2017). 


\section{Supplemental Material}

\section{Analytical perturbation theory in the coupling}

To gain further understanding of the synchronization behavior in the quantum regime, we rederive the synchronization condition (7) explicitly based on perturbation theory in the coupling. To this end we define the perturbation as $\mathcal{L}_{1} \rho=-i[V, \rho]$ and the unperturbed Lindbladian as $\mathcal{L}_{0} \rho=\sum_{j}-i\left[H_{0 j}, \rho\right]+L_{j} \rho$, where we use the definitions from Eq. (4) from the main text and $j=1,2$ indicates the oscillator. The steady-state density operator to first order in the coupling $V$ is then $\rho^{(1)}=-\mathcal{L}_{0}^{-1} \mathcal{L}_{1} \rho^{(0)}$, which we calculate term by term in the following.

The uncoupled density operator $\rho^{(0)}$ is diagonal and for the individual oscillator its (unnormalized) entries $\rho_{m m}^{(0)} \propto$ $\left(\gamma_{+} / \gamma_{-}\right)^{m} \prod_{i=1}^{m} f_{+}(i) / \prod_{i=1}^{m} f_{-}(i)$ are solved via a recursion relation. The superoperator $\mathcal{L}_{0}$ can be decomposed into a term coupling diagonal density matrix elements and a term coupling off-diagonal elements separately. In the off-diagonal subspace, without neglecting any terms, the inverse $\mathcal{L}_{0}^{-1}$ can be obtained by inverting the diagonal so that

$\mathcal{L}_{0}^{-1}\left|m_{1}-1, m_{2}+1\right\rangle\left\langle m_{1}, m_{2}\left|=\lambda_{-}^{-1}\right| m_{1}-1, m_{2}+1\right\rangle\left\langle m_{1}, m_{2}\right|$ with

$$
\begin{aligned}
\lambda_{-} & =-i\left[-\omega_{01}+\omega_{02}+2 K_{1} m_{1}-2 K_{2} m_{2}-2 K_{1}\right] \\
& -\frac{\gamma_{1+}}{2}\left(m_{1} f_{1+}\left(m_{1}\right)+\left(m_{1}+1\right) f_{1+}\left(m_{1}+1\right)\right)-\frac{\gamma_{1-}}{2}\left(\left(m_{1}-1\right) f_{1-}\left(m_{1}-1\right)+m_{1} f_{1-}\left(m_{1}\right)\right) \\
& -\frac{\gamma_{2+}}{2}\left(\left(m_{2}+2\right) f_{2+}\left(m_{2}+2\right)+\left(m_{2}+1\right) f_{2+}\left(m_{2}+1\right)\right)-\frac{\gamma_{2-}}{2}\left(\left(m_{2}+1\right) f_{2-}\left(m_{2}+1\right)+m_{2} f_{2-}\left(m_{2}\right)\right) .
\end{aligned}
$$

As $\mathcal{L}_{1}$ couples only neighboring Fock states, $\rho^{(1)}=-\mathcal{L}_{0}^{-1} \mathcal{L}_{1} \rho^{(0)}$ has nonzero elements only on the minor diagonals, so that the first-order correction for the steady state is

$$
\rho_{m_{1}-1, m_{2}+1 ; m_{1} m_{2}}^{(1)}=-i C \sqrt{m_{1}\left(m_{2}+1\right)} \frac{1}{\lambda_{-}}\left(\rho_{m_{1} m_{2} ; m_{1} m_{2}}^{(0)}-\rho_{m_{1}-1, m_{2}+1 ; m_{1}-1, m_{2}+1}^{(0)}\right)
$$

and a similar expression for $\rho_{m_{1}+1, m_{2}-1 ; m_{1} m_{2}}^{(1)}$. The synchronization measure (6) from the main text can now be written as the sum

$$
S=2\left|\sum_{m_{1}=1, m_{2}=0}^{\infty} \rho_{m_{1}-1, m_{2}+1 ; m_{1}, m_{2}}^{(1)}\right|
$$

of the off-diagonal elements (S2). The analytical expression (S3) for $S$ is a sum over terms (S1) centered around $2 K$ which have widths scaling with $\gamma_{ \pm}$. As an approximate rule, we therefore need $K \gg \gamma_{ \pm}$to resolve the resonances, which is in agreement with the numerical simulation shown in Fig. 3 (d) from the main text. 

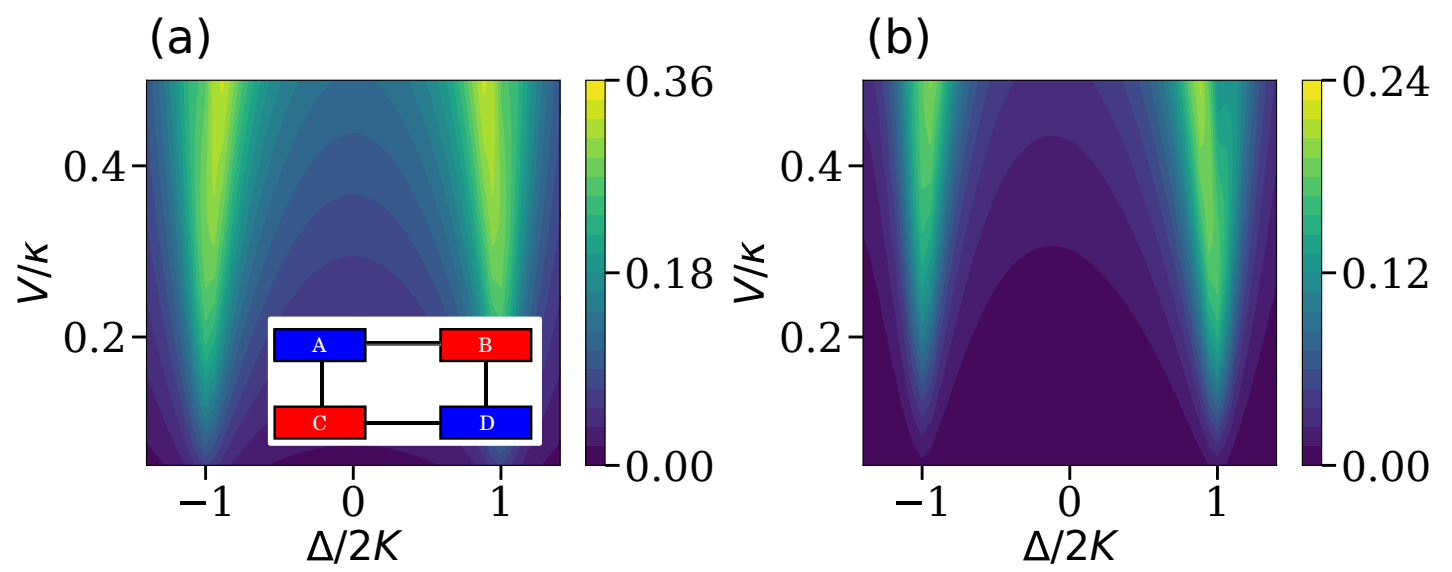

Figure 5. Synchronization measure $S$ for the network of four oscillators depicted in the inset, where neighboring oscillators are coupled with strength $V$. The oscillators $A$ and $D$ are identical and detuned by by $\Delta$ from another identical set oscillators $B$ and $C$. All other parameters are as in Fig. 3 of the main text, i.e. $n_{1}^{+} \equiv 1, n_{j}^{-}=n_{j}^{+}+1, K=10 \gamma, \gamma_{1 \pm}=\gamma_{2 \pm}=\gamma$. Panel (a) shows synchronization as measured by $S$ between a pair of detuned oscillators, e.g. $A$ and $B$. Panel (b) shows synchronization between identical oscillators, e.g. $A$ and $D$. The resonances show that the synchronization blockade between a pair of identical oscillators is lifted by coupling them via a pair of detuned oscillators.

We have also considered a network of four oscillators, where two pairs of identical oscillators are connected in a ring in alternating order. The two species again differ only in a relative detuning $\Delta$ of their frequencies $\omega$. As shown in Fig. 5, synchronization can be mediated via detuned oscillators, but is blocked for identical oscillators due to the quantum synchronization blockade.

\section{Illustration of the effect and minimal model}

In future studies it will be interesting to investigate how the quantum synchronization blockade generalizes to larger networks. It is desirable to restrict the system to a small Hilbert space, to make these simulations numerically feasible. Therefore the individual components of the lattice would ideally be constituted by qubits. However, at least three energy levels per system are required to observe the quantum synchronization blockade effect introduced in this article.

This can be understood from Figure 6, where the origin of the effect is visualized. The crucial diagonal levels are marked in red. These levels are resonant with their neighbors (connected with red lines) for harmonic oscillators. The levels become off-resonant with their neighbors for anharmonic oscillators, resulting in the quantum synchronization blockade for identical oscillators.

A minimal model must keep at least one such red level and its neighbors. The lowest red level with neighbors is $|1,1\rangle$, which is coupled to $|0,2\rangle$ and $|2,0\rangle$. To include these levels, each system must contain at least three levels $(|0\rangle,|1\rangle,|2\rangle)$. As a model must be anharmonic for the red levels to be off-resonant with their neighbors, an anharmonic oscillator with three levels is the minimum model for the effect to be observable. The minimal model is further illustrated in Figure 7. It is an interesting direction to investigate whether also spin systems with three or more levels could be a feasible platform for this effect as well.

The quantum synchronization blockade reported here is unrelated to the observation, that classical anharmonic oscillators with unbalanced losses (resulting in stabilization at different amplitudes) need a finite detuning to compensate for the disbalance to reach maximal synchronization. The suppression of synchronization reported here contradicts the classical expectation, as we have shown. It is also unrelated to the case of two coupled, initially aligned qubits with strongly unbalanced coupling to a thermal quantum bath reported in Ref. 37: Interestingly, the alignment of qubits during time evolution can probe the properties of the bath. Depending on its spectral density, a finite detuning of the unbalanced qubits may favor alignment after transient times before the system equilibrates with the bath. 


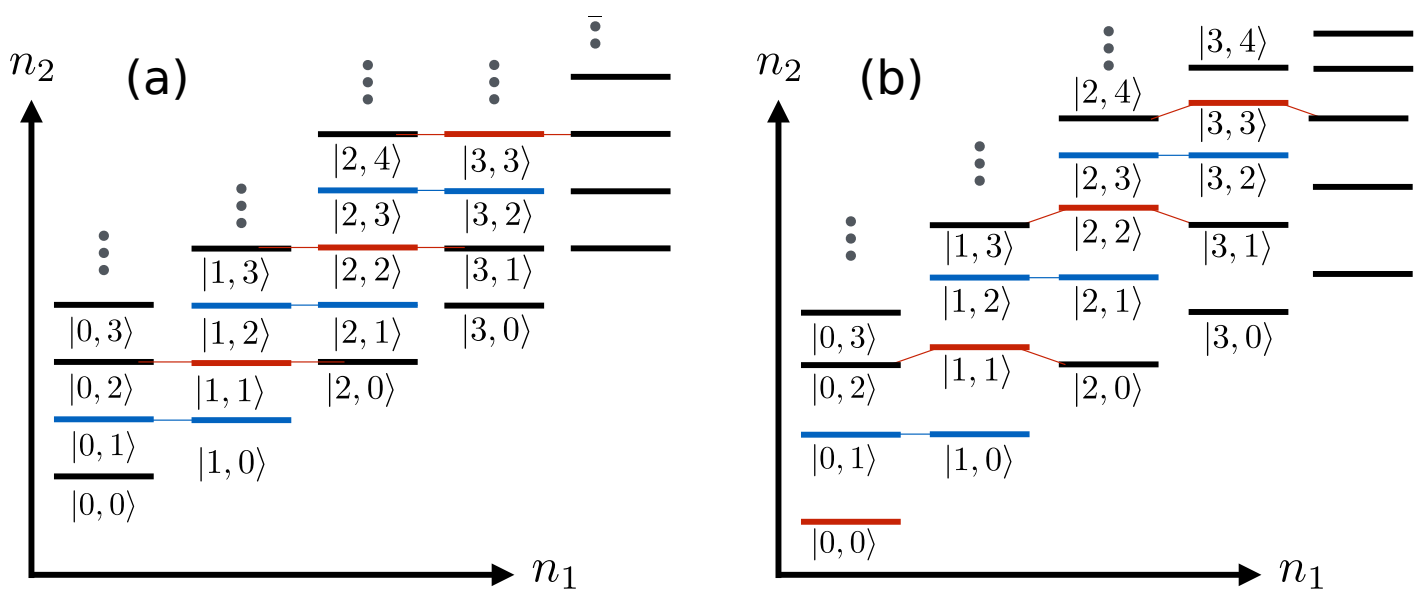

Figure 6. Energy level diagram of two identical oscillators. (a) harmonic oscillators. (b) anharmonic oscillators. The axes $n_{1}$ and $n_{2}$ denote the level of the respective oscillators. The red levels, where both oscillators have the same occupation, are resonant with the two connected adjacent levels in the case of the harmonic oscillator $(K=0)$ depicted on the left. For the nonlinear oscillator on the right, this resonance is lifted. The blue levels corresponding to the condition $\Delta n= \pm 1$ are resonant in both cases and lead to the resonances in Fig. (3) (b) from the main text. For example in the column $n_{1}=2$, the blue levels are $|2,1\rangle$ and $|2,3\rangle$, where $\Delta n= \pm 1$ is fulfilled.

(a)

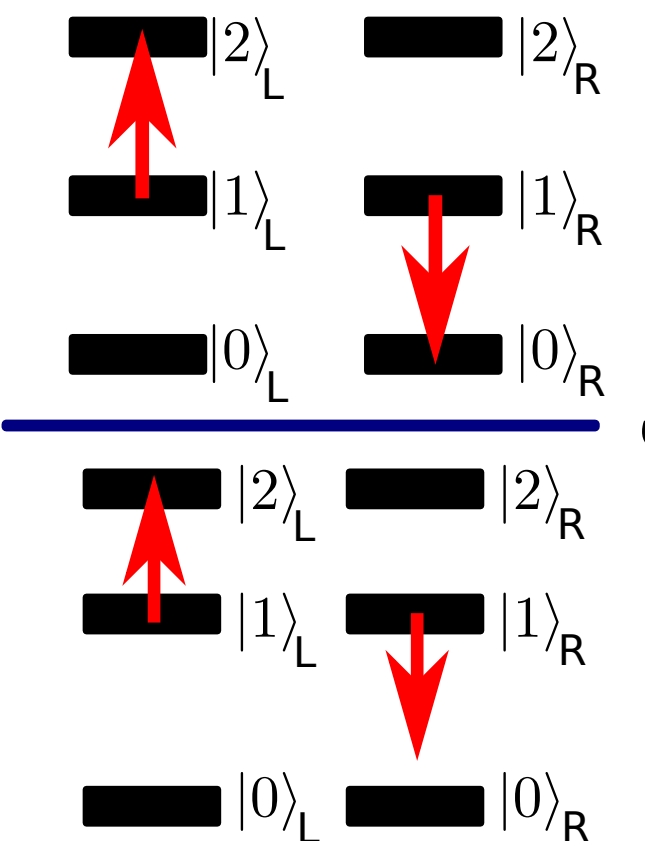

(b)

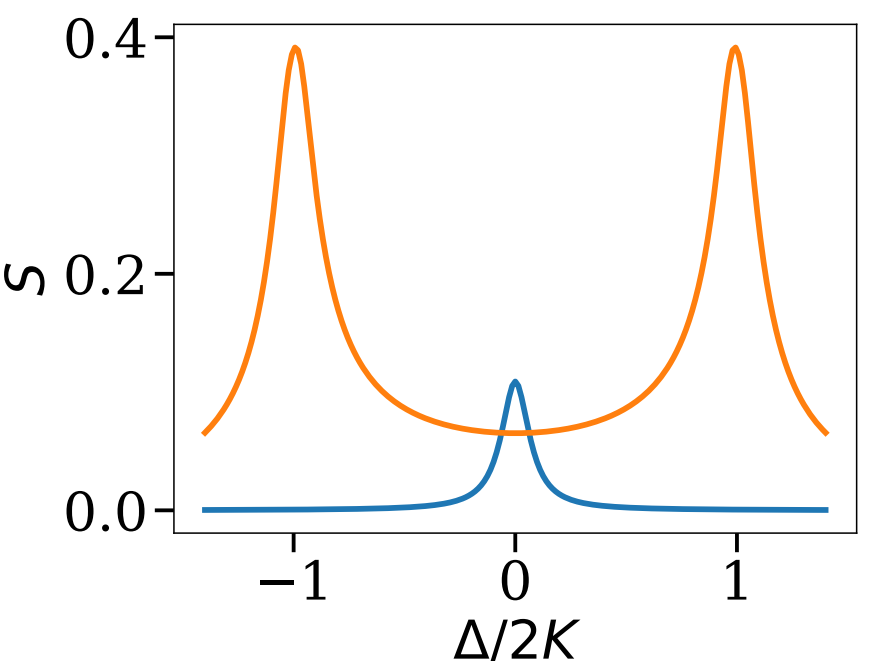

Figure 7. (a) Illustration of the synchronization blockade for the minimal system consisting of two three-level systems with annihilation operators $a_{L}$ and $a_{R}$ indexed by $L$ and $R$ for left and right. Before the interaction is turned on, each system is stabilized to level $|1\rangle$. The interaction $a_{L}^{\dagger} a_{R}$ (red arrows) transfers one excitation from system $a_{R}$ to system $a_{L}$. For the harmonic case depicted in the upper panel, this conserves energy, as both upward and downward transition have the same energy difference. However for the anharmonic case depicted in the lower panel, the left oscillator gains less energy than the right oscillator loses. Therefore interaction is hindered at zero detuning. With a finite detuning $\Delta=2 K$ the process is again resonant. The same argument holds for the reverse process $a_{L} a_{R}^{\dagger}$ at a detuning $\Delta=-2 K$. This is the origin of the two side resonances introduced in Fig. 2(b) of the main text. (b) Synchronization measure $S$ as a function of detuning $\Delta$. For parameters $n_{1}^{+}=n_{2}^{+}=1, \sigma_{1}^{ \pm}=\sigma_{2}^{ \pm}=0.2, V=0.8 \gamma$ the resonance curve of a harmonic oscillator (blue line) with $K=0$ is compared to an anharmonic oscillator (orange line) with $K=3 \gamma / \sigma$. In both cases the oscillators are restricted to the lowest three levels. While the harmonic oscillator confirms the classical expectation of maximal synchronization on resonance, the anharmonic oscillator's resonances are shifted as explained in (a). We also numerically checked that in the case of two-level systems $S=0$ for all $\Delta$, as the effect is not applicable to qubits. 